\title{
Time-Resolved Radiation Chemistry: Photoelectron Imaging of Transient Negative lons of Nucleobases
}

\author{
Margaret A. Yandell, ${ }^{\dagger}$ Sarah B. King, ${ }^{\dagger}$ and Daniel M. Neumark ${ }^{*}, \dagger+$ \\ ${ }^{\dagger}$ Department of Chemistry, University of California, Berkeley, California 94720, United States \\ ${ }^{\ddagger}$ Chemical Sciences Division, Lawrence Berkeley National Laboratory, Berkeley, California 94720, United States
}

ABSTRACT: Time-resolved photoelectron imaging has been utilized to probe the energetics and dynamics of the transient negative ion of the nucleobase uracil. This species was created through charge transfer from an iodide anion within a binary iodide-uracil complex using a UV pump pulse; the ensuing dynamics were followed by photodetachment with a near-IR probe pulse. The photoelectron spectra show two time-dependent features, one from probe-induced photodetachment of the transient anion state and another from very low energy electron signal attributed to autodetachment. The transient anion was observed to decay biexponentially with time constants of hundreds of femtoseconds and tens of picoseconds, depending on the excitation energy. These dynamics are interpreted in terms of autodetachment from the initially excited state and a second, longer-lived species relaxed by iodine loss. Hydrogen loss from the $\mathrm{N} 1$ position may also occur in parallel.

$\mathrm{T}$ he observation that low-energy electrons can lead to DNA and RNA strand cleavage via temporary negative ion states $^{1}$ has motivated numerous studies of nucleic acid constituents. Gas-phase studies of DNA and RNA building blocks, including individual nucleobases, nucleosides, and nucleotides, have sought to provide insight into the mechanisms of this radiation damage. ${ }^{2}$ Transient anion states of nucleobases have been posited to play a major role in DNA mutagenesis, perhaps via a charge-transfer process from an initially charged nucleobase moiety to a sugar-phosphate bond. ${ }^{3,4}$ In this work, we explore the dynamics of transient anion states of uracil via time-resolved photoelectron (TRPE) imaging $^{5}$ of an iodide-uracil binary complex.

The interaction of excess electrons with uracil and other nucleobases has been studied in the gas phase using low-energy electron scattering, ${ }^{6,7}$ negative ion photoelectron spectroscopy (PES), ${ }^{8-10}$ and Rydberg electron transfer (RET). ${ }^{11}$ Total electron scattering cross section measurements showed structure below $2 \mathrm{eV}$ associated with unoccupied $\pi^{*}$ orbitals of uracil, ${ }^{6}$ while dissociative electron attachment (DEA) studies showed that hydrogen atom loss from the N1 position in the transient negative ion $\mathrm{U}^{*-}$ occurs at collision energies as low as $0.7 \mathrm{eV}^{6,7,12-14}$

The nature of the uracil anion has been directly probed in PES and RET studies. PES experiments have measured the binding energy of the dipole-bound species as $\sim 90 \mathrm{meV}^{8,9}$ and estimate that valence anions of uracil bind excess electrons by tens to hundreds of meV. ${ }^{9-11}$ These species are clearly distinguishable in photoelectron (PE) spectra, as dipolebound states consist of narrow features with low electron binding energies, reflecting the similarity between the anion and neutral geometries, while valence-bound anions have characteristically broader features. ${ }^{15}$ Only dipole-bound anions of uracil have been observed using conventional ion generation methods, ${ }^{8-10}$ but the uracil anion can transform from a dipolebound state to a valence-bound state upon complexation with one Xe atom or water molecule., 15

Though DEA and single-photon PES experiments probe aspects of the uracil anion, neither provides a complete picture of its energetics and dynamics. Here we describe a novel approach to investigating the time-resolved dynamics of electron attachment to uracil based on photoinitiation of intracluster charge transfer in a binary halide-nucleobase complex, $\mathrm{I}^{-} \cdot \mathrm{U}$, to form a transient negative ion (TNI) of uracil, $\mathrm{I} \cdots \mathrm{U}^{*-}$ :

$$
\mathrm{I}^{-} \cdot \mathrm{U} \stackrel{h \nu_{\text {pump }}}{\longrightarrow} \mathrm{I} \cdots \mathrm{U}^{*^{-}} \rightarrow ?
$$

Upon the application of a femtosecond UV pump pulse at or above the threshold for direct detachment of the iodide-uracil complex, the excess electron is ejected with low kinetic energy and can interact with the uracil moiety to form a TNI, in analogy to DEA studies. A second time-delayed femtosecond pulse at $790 \mathrm{~nm}(1.57 \mathrm{eV})$ detaches the electron. The resulting electron kinetic energy (eKE) distribution is measured using photoelectron imaging, thereby probing the lifetime and decay dynamics of the TNI.

Iodide-uracil clusters were prepared by flowing argon gas over a methyl iodide reservoir into an Even-Lavie valve ${ }^{16}$ containing uracil heated to $205{ }^{\circ} \mathrm{C}$ and operating at a pulse repetition rate of $500 \mathrm{~Hz}$. Further details of the femtosecond TRPE spectrometer have been described elsewhere. ${ }^{17-19}$

Figure 1 shows one-photon PE spectra of the iodide-uracil binary complex recorded at excitation energies of 4.21-5.31 eV and plotted as a function of electron binding energy (defined as $\mathrm{eBE}=h \nu-\mathrm{eKE})$. Two main features are apparent in the spectra for all five excitation energies: an energy-invariant peak A centered at $4.11 \mathrm{eV}$, representing the vertical detachment energy (defined as VDE $=h \nu-\mathrm{eKE}_{\max }$ ) of $\mathrm{I}^{-} \cdot \mathrm{U}$, and a feature $\mathrm{C}$ appearing at approximately $\mathrm{eBE}=h \nu$, corresponding to electron signal at nearly zero kinetic energy. The spectrum at $h \nu=5.31 \mathrm{eV}$ also shows an additional direct detachment feature $\mathrm{B}$ arising from the ${ }^{2} \mathrm{P}_{1 / 2}$ spin-orbit state of complexed iodine that is inaccessible at lower excitation energies. The VDE

Received: December 19, 2012

Published: January 25, 2013 


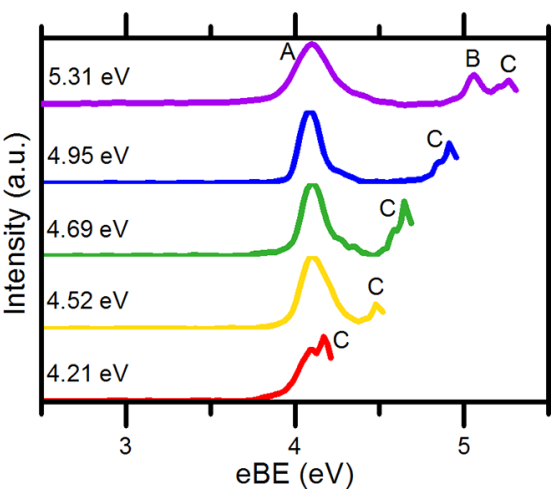

Figure 1. One-photon PE spectra of the iodide-uracil binary complex at several excitation energies.

obtained from feature A indicates a solvent shift of $1.05 \mathrm{eV}$ with respect to bare iodide. This shift is noticeably larger than those seen for binary complexes of iodide with tetrahydrofuran $(0.25$ $\mathrm{eV}){ }^{20}$ water $(0.45 \mathrm{eV}),{ }^{21}$ and aniline $(0.53 \mathrm{eV}) .^{22}$

Figure 2 displays TRPE spectra plotted against eKE for the binary cluster excited at $4.69 \mathrm{eV}$ and probed at $1.57 \mathrm{eV}$.

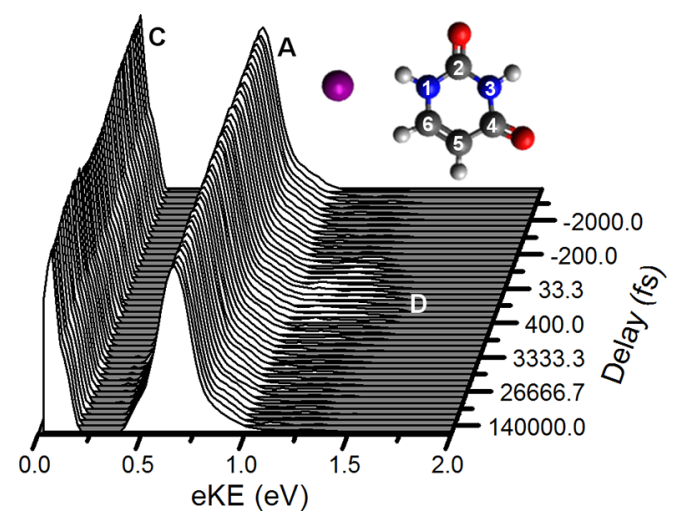

Figure 2. TRPE spectrum of the $\mathrm{I}^{-} \cdot \mathrm{U}$ complex excited at $4.69 \mathrm{eV}$ and probed at $1.57 \mathrm{eV}$. The inset shows the optimized iodide-uracil cluster geometry.

Features $\mathrm{A}$ and $\mathrm{C}$ at $0.6 \mathrm{eV}$ and near zero kinetic energy, respectively, are apparent at every pump-probe delay $(\Delta t)$, while a third, broad, low intensity feature D appears between 1.0-1.6 eV at $\Delta t=0$ and survives for several picoseconds. Similar spectra were observed for excitation energies of 4.32 $5.02 \mathrm{eV}$, but because of a low signal-to-noise ratio outside the central portion of this energy range, only the results obtained for pump energies of 4.69-4.90 eV will be discussed here.

The integrated intensities of features $\mathrm{C}$ and $\mathrm{D}$ (Figure 3 ) reveal complementary yet differing dynamics. At $\Delta t=0$, the transient feature $\mathrm{D}$ appears and feature $\mathrm{C}$ is depleted. The transient feature then exhibits biexponential decay; Table 1 shows time constants derived by fitting its dynamics to a biexponential function convoluted with a Gaussian function ${ }^{23}$ with a full width at half-maximum of $150 \mathrm{fs}$. We found short decay time constants $\left(\tau_{1}\right)$ of $300-600 \mathrm{fs}$ and long decay time constants $\left(\tau_{2}\right)$ of 10-50 ps. Both time constants were observed to decrease with increasing excitation energy. The depletion and initial recovery of feature $\mathrm{C}$ mirror the early time dynamics of feature $\mathrm{D}$, however feature $\mathrm{C}$ overshoots its initial intensity within $1-2$ ps and continues to rise for $\sim 20$ ps before decaying over 50-100 ps.
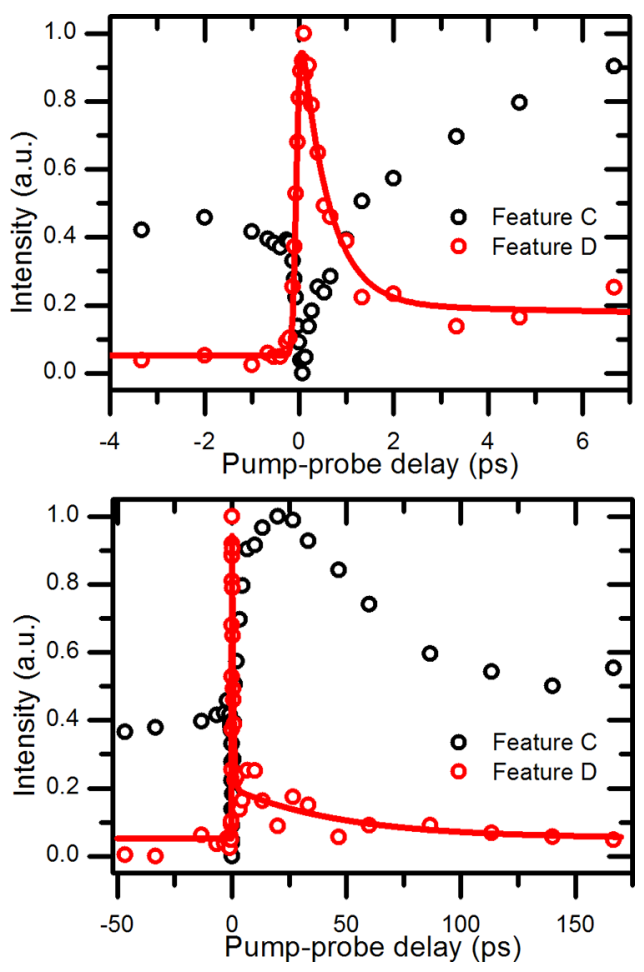

Figure 3. Population dynamics of features $C$ and $D$ for the $\mathrm{I}^{-} \cdot \mathrm{U}$ complex upon excitation at $4.69 \mathrm{eV}$. The top panel demonstrates the concomitant decay and rise in the respective features.

Table 1. Time Constants for Decay of the Transient Species ${ }^{a}$

$\begin{array}{ccc}\text { excitation energy }(\mathrm{eV}) & \tau_{1}(\mathrm{fs}) & \tau_{2}(\mathrm{ps}) \\ 4.90 & 300 \pm 50 & 12 \pm 6 \\ 4.79 & 390 \pm 80 & 37 \pm 20 \\ 4.69 & 620 \pm 50 & 52 \pm 20\end{array}$

${ }^{a}$ Values are reported as variance-weighted means and their associated errors.

Geometry optimizations of the binary cluster were carried out using Gaussian $09^{24}$ at the MP2/aug-cc-pVDZ level with an aug-cc-pVDZ-pp pseudopotential ${ }^{25}$ for iodide. The Figure 2 inset shows the optimized structure, which has $C_{s}$ symmetry: the iodide anion resides between the hydrogen bound to N1 and the neighboring vinyl hydrogen near the positive pole of the $\sim 5 \mathrm{D}$ permanent dipole moment of uracil. ${ }^{6,26}$ Relative to the previously calculated equilibrium geometry of neutral uracil, ${ }^{27}$ the $\mathrm{N} 1-\mathrm{H}$ and $\mathrm{C} 5-\mathrm{C} 6$ bonds of the iodide-complexed species are slightly elongated and the hydrogen atoms bound to $\mathrm{N} 1$ and C6 angle inward by several degrees. Subsequent energy optimizations at the CCSD level found a VDE of $4.13 \mathrm{eV}$, consistent with experimental results and previous density functional theory calculations. ${ }^{28}$

Comparison between measured TRPE spectra for the $\mathrm{I}^{-} \cdot \mathrm{U}$ cluster and those for iodide-containing solvent clusters ${ }^{19,29-31}$ suggests that feature D arises from probe-induced photodetachment from the I... U*- state created by the pump pulse, whereas feature $\mathrm{C}$ at low eKE represents vibrational autodetachment from this species:

$$
\begin{array}{r}
\mathrm{I}^{-} \cdot \mathrm{U} \stackrel{h \nu_{\text {pump }}}{\longrightarrow} \mathrm{I} \cdots \mathrm{U}^{*^{-}} \stackrel{h \nu_{\text {probe }}}{\longrightarrow} \mathrm{I} \cdots \mathrm{U}+\mathrm{e}^{-} \quad(\text { feature } \mathrm{D}) \\
\stackrel{k_{1}}{\rightarrow} \mathrm{I} \cdots \mathrm{U}+\mathrm{e}^{-} \quad(\text { feature } \mathrm{C})
\end{array}
$$


However, as discussed below, eq 2 represents only a partial picture of the $\mathrm{I} \cdots \mathrm{U}^{*-}$ decay dynamics.

The cluster excited state I... $\mathrm{U}^{*-}$ is formed by transfer of the excess electron from iodide to the uracil molecule to create an excited uracil anion perturbed by an iodine atom. The broadness of the corresponding transient feature $\mathrm{D}$ in the photoelectron spectrum is characteristic of a valence-bound state. Moreover, the VDE of feature $\mathrm{D}$ lies between 0 and 0.6 $\mathrm{eV}$, close to the calculated VDE of $0.6 \mathrm{eV}$ for the valence-bound state of the uracil anion formed by adding an electron to the low-lying, unoccupied $\pi^{*}$ orbital of uracil. ${ }^{27}$ It thus appears that the TNI formed by charge transfer from the iodide anion is this valence-bound species rather than a dipole-bound state. The presence of the iodine atom may suppress the initial formation of a dipole-bound state as it resides within the region of maximum electron density for the dipole-bound state of the bare anion. ${ }^{14}$

Our experiment can be considered in terms of intracluster electron scattering: the electron produced by photodetachment from the iodine moiety will either be ejected into free space, resulting in the direct detachment features $\mathrm{A}$ and $\mathrm{B}$, or undergo indirect scattering from the uracil molecule to form the TNI, from which the time-dependent features $\mathrm{C}$ and $\mathrm{D}$ derive. The effective electron collision energy is then given by $E_{c}=h \nu_{\text {pump }}-$ VDE, where the VDE of the $\mathrm{I}^{-} \cdot \mathrm{U}$ cluster is $4.11 \mathrm{eV}$ as determined from feature $A$ in Figure 1 . Thus, $E_{\mathrm{c}}$ varies from 0.1 to $1.2 \mathrm{eV}$ over the range of excitation energies considered and includes the energy range where electron scattering experiments and theoretical studies show attachment to the uracil $\pi^{*}$ orbital. $^{32,33}$

Direct absorption of the UV photon by the uracil moiety itself is possible, as the excitation energies utilized here fall on the low-energy side of its $\pi \rightarrow \pi^{*}$ absorption band. ${ }^{34,35}$ This process would lead to a complex comprised of iodide and an electronically excited uracil molecule, U*. Photodetachment of such a species by the IR probe photon would not be feasible. However, if charge transfer from iodide into the singly occupied $\pi$ orbital of $\mathrm{U}^{*}$ could occur within this complex, the same $\mathrm{I} \cdots \mathrm{U}^{*-}$ final state discussed above would result. This process would most likely lead to an observable delay in the buildup of feature $\mathrm{D}$, which is inconsistent with the nearly instantaneous rise observed for this signal. Moreover, the 10-fold enhancement of the absorption cross section for uracil between 4.5 and $5.3 \mathrm{eV}$ would be reflected by a substantial increase in the autodetachment yield relative to the direct detachment yield in the one-photon PE spectra, but no such trend was observed. It thus appears that the results presented here do not arise through this mechanism.

We next consider the possible decay pathways for the TNI. The complementary early time dynamics of the transient and autodetachment features imply that the $\mathrm{I} \cdots \mathrm{U}^{*-}$ state created by the pump pulse decays at least in part via autodetachment, as shown in eq 2 . While the canonical tautomer of neutral uracil is planar, ${ }^{27}$ the $\pi^{*}$ valence-bound anion state has a nonplanar, puckered structure, ${ }^{10,27,28}$ so electron attachment into the $\pi^{*}$ orbital would result in significant vibrational excitation in the anion state, which would then be metastable with respect to autodetachment. We observed the general trend that the decay rate of the TNI increases with excitation energy, which translates into increased vibrational energy in the TNI. This behavior is expected for a statistical process such as vibrational autodetachment, ${ }^{19}$ which has also been identified as the primary decay mechanism for other iodide-containing cluster systems. ${ }^{19,29-31}$ However, the results for $\mathrm{I}^{-} \cdot \mathrm{U}$ differ from these previous experiments because (i) the transient feature D exhibits biexponential rather than monoexponential decay and (ii) the autodetachment feature $\mathrm{C}$ does not simply mirror the time dependence of feature $\mathrm{D}$. We therefore must consider other decay mechanisms for the TNI that could occur in addition to autodetachment.

The observation of biexponential decay for the transient feature implies that the TNI decays not only by autodetachment but also to another state with a longer lifetime. The TRPE spectra do not display an accompanying shift in VDE for the transient feature $\mathrm{D}$, indicating that the second state has a VDE similar to that of the initial state. A plausible mechanism involves loss of atomic iodine followed by autodetachment in addition to the dynamics in eq 2 :

$$
\begin{aligned}
\mathrm{I}^{-} \cdot \mathrm{U} \stackrel{h \nu_{\text {pump }}}{\rightarrow} \mathrm{I} \cdots \mathrm{U}^{*^{-}} \stackrel{k_{2}}{\rightarrow} \mathrm{I}+\mathrm{U}^{*^{-}} \stackrel{h \nu_{\text {probe }}}{\longrightarrow} \mathrm{I}+\mathrm{U}+\mathrm{e}^{-} \\
\stackrel{k_{3}}{\rightarrow} \mathrm{I}+\mathrm{U}+\mathrm{e}^{-}
\end{aligned}
$$

In this case, $\tau_{1}=1 /\left(k_{1}+k_{2}\right)$ and $\tau_{2}=1 / k_{3}$. The binding energy of the neutral iodine atom to the TNI in the initially prepared $\mathrm{I} \cdots \mathrm{U}^{*-}$ state is expected to be on the order of $50 \mathrm{meV},{ }^{29,36}$ so iodine loss should be feasible due to the vibrational excitation of the initial TNI. The resulting anionic uracil fragment, $\mathrm{U}^{*^{-}}$, would be a valence-bound state with a VDE similar to that of the initial TNI. Thus, probe-induced detachment would contribute to feature $\mathrm{D}$, however the $\mathrm{U}^{*-}$ state should autodetach more slowly than the initial state because of its reduced vibrational energy.

Another channel to consider is hydrogen atom loss from the uracil N1 position to form $(\mathrm{U}-\mathrm{H})^{-6,7,12-14,37}$ Electron transmission spectroscopy and DEA studies have estimated the threshold energy for this channel to be at most $0.8 \mathrm{eV} .^{6,12}$ In our experiment, the analogous process could occur via

$$
\mathrm{I}^{-} \cdot \mathrm{U} \stackrel{h \nu_{\text {pump }}}{\longrightarrow} \mathrm{I} \cdots \mathrm{U}^{*^{-}} \rightarrow \mathrm{I} \cdots(\mathrm{U}-\mathrm{H})^{-}+\mathrm{H}
$$

The previously defined effective electron collision energy, $E_{c}$ exceeds the threshold for DEA at excitation energies of $4.9 \mathrm{eV}$ or lower. These values are close to the calculated threshold of $4.6 \mathrm{eV}$ for $\mathrm{H}$ atom loss from $\mathrm{I}^{-} \cdot \mathrm{U}^{28}$ Thus, the $\mathrm{H}$ atom loss channel likely opens in the range of excitation energies used herein and $\mathrm{H}$ loss may occur in parallel with autodetachment and iodine loss. However, direct observation of this process is not possible because the binding energy of the $\mathrm{I} \cdots(\mathrm{U}-\mathrm{H})^{-}$ species is expected to exceed $3 \mathrm{eV}^{38-41}$ a value well above the IR probe energy.

Comparison between the transient and autodetachment signals in Figure 3 reveals that the "overshoot" and decay of the autodetachment feature $\mathrm{C}$ occur during the longer-time decay of feature D. Assuming that our assignment of this longer-time decay to autodetachment from vibrationally excited $\mathrm{U}^{*^{-}}$(eq 3 ) is correct, the time dependence of feature $\mathrm{C}$ may indicate that the probe laser enhances the autodetachment signal from this species in addition to detaching it directly. Such a process could occur if the probe laser were resonant with an electronic transition in the $\mathrm{U}^{*-}$ fragment; a similar mechanism has been invoked to explain probe-enhanced autoionization in timeresolved experiments on He droplets. ${ }^{42}$ Further consideration is needed to confirm this interpretation of the dynamics of feature C.

The experiments described herein represent the first direct measurements of the lifetime of the transient negative ion of 
uracil. The insight into the temporary uracil anion state gained in this time-resolved photoelectron spectroscopy study demonstrates the strong link between TRPES and DEA experiments and establishes TRPES as a powerful method for probing the energetics and dynamics of electron attachment to nucleobases. Thus, we have shown that time-resolved radiation chemistry can be used to examine transient negative ions of nucleic acid building blocks in the gas phase from their formation to their decay via fragmentation or relaxation.

\section{AUTHOR INFORMATION}

\section{Corresponding Author}

dneumark@berkeley.edu

\section{Notes}

The authors declare no competing financial interest.

\section{ACKNOWLEDGMENTS}

This research was supported by the National Science Foundation (NSF) under Grant CHE-1011819. M.A.Y. and S.B.K. acknowledge funding from NSF Graduate Research Fellowships.

\section{REFERENCES}

(1) Alizadeh, E.; Sanche, L. Chem. Rev. 2012, 112, 5578.

(2) Hudock, H. R.; Levine, B. G.; Thompson, A. L.; Satzger, H.; Townsend, D.; Gador, N.; Ullrich, S.; Stolow, A.; Martinez, T. J. J. Phys. Chem. A 2007, 111, 8500.

(3) Simons, J. Acc. Chem. Res. 2006, 39, 772.

(4) Gu, J.; Leszczynski, J.; Schaefer, H. F., III. Chem. Rev. 2012, 112, 5603.

(5) Stolow, A.; Bragg, A. E.; Neumark, D. M. Chem. Rev. 2004, 104, 1719.

(6) Scheer, A. M.; Aflatooni, K.; Gallup, G. A.; Burrow, P. D. Phys. Rev. Lett. 2004, 92, No. 068102.

(7) Ptasinska, S.; Denifl, S.; Scheier, P.; Illenberger, E.; Mark, T. D. Angew. Chem., Int. Ed. 2005, 44, 6941.

(8) Hendricks, J. H.; Lyapustina, S. A.; deClercq, H. L.; Snodgrass, J. T.; Bowen, K. H. J. Chem. Phys. 1996, 104, 7788.

(9) Schiedt, J.; Weinkauf, R.; Neumark, D. M.; Schlag, E. W. Chem. Phys. 1998, 239, 511.

(10) Bachorz, R. A.; Klopper, W.; Gutowski, M.; Li, X.; Bowen, K. H. J. Chem. Phys. 2008, 129, No. 054309.

(11) Desfrancois, C.; AbdoulCarime, H.; Schermann, J. P. J. Chem. Phys. 1996, 104, 7792.

(12) Hanel, G.; Gstir, B.; Denifl, S.; Scheier, P.; Probst, M.; Farizon, B.; Farizon, M.; Illenberger, E.; Mark, T. D. Phys. Rev. Lett. 2003, 90, 4.

(13) Li, X. F.; Sanche, L.; Sevilla, M. D. J. Phys. Chem. B 2004, 108, 5472.

(14) Burrow, P. D.; Gallup, G. A.; Scheer, A. M.; Denifl, S.; Ptasinska, S.; Mark, T.; Scheier, P. J. Chem. Phys. 2006, 124, No. 124310.

(15) Hendricks, J. H.; Lyapustina, S. A.; de Clercq, H. L.; Bowen, K. H. J. Chem. Phys. 1998, 108, 8.

(16) Even, U.; Jortner, J.; Noy, D.; Lavie, N.; Cossart-Magos, C. J. Chem. Phys. 2000, 112, 8068.

(17) Davis, A. V.; Wester, R.; Bragg, A. E.; Neumark, D. M. J. Chem. Phys. 2003, 118, 999.

(18) Bragg, A. E.; Verlet, J. R. R.; Kammrath, A.; Cheshnovsky, O.; Neumark, D. M. J. Am. Chem. Soc. 2005, 127, 15283.

(19) Yandell, M. A.; Young, R. M.; King, S. B.; Neumark, D. M. J. Phys. Chem. A 2012, 116, 2750.

(20) Young, R. M.; Azar, R. J.; Yandell, M. A.; King, S. B.; HeadGordon, M.; Neumark, D. M. Mol. Phys. 2012, 110, 1787.

(21) Markovich, G.; Pollack, S.; Giniger, R.; Cheshnovsky, O. J. Chem. Phys. 1994, 101, 9344.

(22) Bowen, M. S.; Becucci, M.; Continetti, R. E. J. Chem. Phys. 2006, 125, No. 133309.
(23) Pedersen, S.; Zewail, A. H. Mol. Phys. 1996, 89, 1455.

(24) Frisch, M. J.; Trucks, G. W.; Schlegel, H. B.; Scuseria, G. E.; Robb, M. A.; Cheeseman, J. R.; Scalmani, G.; Barone, V.; Mennucci, B.; Petersson, G. A.; Nakatsuji, H.; Caricato, M.; Li, X.; Hratchian, H. P.; Izmaylov, A. F; Bloino, J.; Zheng, G.; Sonnenberg, J. L.; Hada, M.; Ehara, M.; Toyota, K.; Fukuda, R.; Hasegawa, J.; Ishida, M.; Nakajima, T.; Honda, Y.; Kitao, O.; Nakai, H.; Vreven, T.; Montgomery, J. A., Jr.; Peralta, J. E.; Ogliaro, F.; Bearpark, M.; Heyd, J. J.; Brothers, E.; Kudin, K. N.; Staroverov, V. N.; Kobayashi, R.; Normand, J.; Raghavachari, K.; Rendell, A.; Burant, J. C.; Iyengar, S. S.; Tomasi, J.; Cossi, M.; Rega, N.; Millam, J. M.; Klene, M.; Knox, J. E.; Cross, J. B.; Bakken, V.; Adamo, C.; Jaramillo, J.; Gomperts, R.; Stratmann, R. E.; Yazyev, O.; Austin, A. J.; Cammi, R.; Pomelli, C.; Ochterski, J. W.; Martin, R. L.; Morokuma, K.; Zakrzewski, V. G.; Voth, G. A.; Salvador, P.; Dannenberg, J. J.; Dapprich, S.; Daniels, A. D.; Farkas, Ö.; Foresman, J. B.; Ortiz, J. V.; Cioslowski, J.; Fox, D. J. Gaussian 09, Revision C.01; Gaussian, Inc.: Wallingford, CT, 2009.

(25) Peterson, K. A.; Shepler, B. C.; Figgen, D.; Stoll, H. J. Phys. Chem. A 2006, 110, 13877.

(26) Oyler, N. A.; Adamowicz, L. J. Phys. Chem. 1993, 97, 11122.

(27) Bachorz, R. A.; Klopper, W.; Gutowski, M. J. Chem. Phys. 2007, 126, No. 085101.

(28) Martínez, A.; Dolgounitcheva, O.; Zakrzewski, V. G.; Ortiz, J. V. J. Phys. Chem. A 2008, 112, 10399.

(29) Kammrath, A.; Verlet, J. R. R.; Bragg, A. E.; Griffin, G. B.; Neumark, D. M. J. Phys. Chem. A 2005, 109, 11475.

(30) Ehrler, O. T.; Griffin, G. B.; Young, R. M.; Neumark, D. M. J. Phys. Chem. B 2009, 113, 4031.

(31) Young, R. M.; Yandell, M. A.; Neumark, D. M. J. Chem. Phys. 2011, 134, No. 124311.

(32) Aflatooni, K.; Gallup, G. A.; Burrow, P. D. J. Phys. Chem. A 1998, 102, 6205.

(33) Winstead, C.; McKoy, V. J. Chem. Phys. 2006, 125, No. 174304.

(34) Clark, L. B.; Peschel, G. G.; Tinoco, I. J. Phys. Chem. 1965, 69, 3615.

(35) Barbatti, M.; Aquino, A. J. A.; Lischka, H. Phys. Chem. Chem. Phys. 2010, 12, 4959.

(36) Chen, H. Y.; Sheu, W. S. Chem. Phys. Lett. 2001, 335, 475.

(37) Denifl, S.; Ptasinska, S.; Hanel, G.; Gstir, B.; Probst, M.; Scheier, P.; Mark, T. D. J. Chem. Phys. 2004, 120, 6557.

(38) Profeta, L. T. M.; Larkin, J. D.; Schaefer, H. F., III. Mol. Phys. 2003, 101, 3277.

(39) Miller, T. M.; Arnold, S. T.; Viggiano, A. A.; Stevens Miller, A. E. J. Phys. Chem. A 2004, 108, 3439.

(40) Vazquez, M. V.; Martinez, A.; Dolgounitcheva, O.; Ortiz, J. V. J. Phys. Chem. A 2006, 110, 11174.

(41) Parsons, B. F.; Sheehan, S. M.; Yen, T. A.; Neumark, D. M.; Wehres, N.; Weinkauf, R. Phys. Chem. Chem. Phys. 2007, 9, 3291.

(42) Kornilov, O.; Buenermann, O.; Haxton, D. J.; Leone, S. R.; Neumark, D. M.; Gessner, O. J. Phys. Chem. A 2011, 115, 7891. 